\title{
Asymptotic Analysis and Error Estimate for Rosenau-Burgers Equation
}

\author{
Jun Zhang $\mathbb{D}^{1}{ }^{1}$ Zixin Liu $\left(\mathbb{D},{ }^{2}\right.$ Fubiao Lin, ${ }^{2}$ and Jianjun Jiao ${ }^{2}$ \\ ${ }^{1}$ Computational Mathematics Research Center, Guizhou University of Finance and Economics, Guiyang, Guizhou 550025, China \\ ${ }^{2}$ School of Mathematics and Statistical, Guizhou University of Finance and Economics, Guiyang, Guizhou 550025, China \\ Correspondence should be addressed to Zixin Liu; xinxin905@163.com
}

Received 18 March 2019; Revised 23 April 2019; Accepted 21 May 2019; Published 23 June 2019

Academic Editor: Vincenzo Vespri

Copyright (c) 2019 Jun Zhang et al. This is an open access article distributed under the Creative Commons Attribution License, which permits unrestricted use, distribution, and reproduction in any medium, provided the original work is properly cited.

In this work, the asymptotic stability result for Rosenau-Burgers equation is established, under appropriate assumptions on steady state eigenvalue problem and the forcing function. In addition, we propose and analyze a linearized numerical method for solving this nonlinear Rosenau-Burgers equation. We prove that the numerical scheme is unconditionally stable, and the error estimate shows that the numerical method is in the order of $\mathcal{O}\left(\Delta t^{2}+N^{2-m}\right)$, where $\Delta t, N$, and $m$ are, respectively, step of time, polynomial degree, and regularity of $u$. Numerical examples are illustrated to verify the theoretical results.

\section{Introduction}

In the research of dynamic dense discrete system, it was shown that the KdV equation can not completely describe the interaction between waves and waves, and in order to overcome the shortcoming of this $\mathrm{KdV}$ equation, Rosenau $[1,2]$ proposed the following Rosenau equation:

$$
u_{t}+u_{x x x x t}+u_{x}+u u_{x}=0
$$

For further consideration of dissipation in dynamic system, the term $-\alpha u_{x x}$ is included in the above equatoin. The resulting new equation is called the Rosenau-Burgers equation:

$$
u_{t}+u_{x x x x t}-\alpha u_{x x}+u_{x}+u u_{x}=0 .
$$

Park [3] discussed existence and uniqueness properties for the Rosenau-Burgers equation. Mei \& Liu [4-7] studied the large-time behavior of the solution for the RosenauBurgers equation, and they obtained some estimates for the asymptotic solution. Kinami [8] \& Mei [9] considered the asymptotic behavior of solution for Benjamin-BonaMahony-Burgers equation. They proved that the solution asymptotically converges to 0 .

Besides the theoretical analysis, some recent contributions focus on using numerical methods to approximate the solutions of Rosenau-Burgers equation. Chung [10] \& Sank [11] introduced finite element Galerkin method for solving a Rosenau equation. They obtained the existence and uniqueness of solutions and the error estimates of the solutions are also discussed. Chung [12], Omrani [13], and Feng [14] presented a conservative finite difference scheme for solving Rosenau equation. Convergence, stability, and error estimate of full discrete scheme are also proved. Manickam et al. [15] applied orthogonal cubic spline collocation method to approximate Rosenau equation, and the error estimates are obtained in both $L^{2}$ norm and $L^{\infty}$ norm.

In this paper, we consider the following Rosenau-Burgers equation:

$$
\begin{aligned}
u_{t}+u_{x x x x t}-\alpha u_{x x}+u_{x}+u u_{x} & =f(x, t), \\
& \\
u(x, 0) & =u_{0}(x), \\
u(x, t) & =u(x+2 L, t),
\end{aligned}
$$

where $\alpha>0$.

In this article, the convergence of the Rosenau-Burgers equation to its steady-state problem is discussed, under the assumption that the corresponding linearized steady-state eigenvalue problem has a positive minimum eigenvalue and 
appropriate condition on the forcing function. Moreover, we present numerical method to solve Rosenau-Burgers equation, and the proposed scheme is performed by combining Crank-Nicolson approach in time and Fourier-spectral in space. Our rigorous analysis result shows that the scheme is unconditionally stable, and the numerical method leads to second order in time and spectral accuracy in space.

The rest of the article is organized in the following way. Section 2 will study asymptotic behaviour of RosenauBurgers equation. In Section 3, we will discuss stability and error estimate for the full discrete scheme. In Section 4, we present some numerical experiments to illustrate the validity of the numerical method. The conclusions of this paper are given in Section 5.

\section{Asymptotic Behaviour of Rosenau-Burgers Equation}

In this section, we will investigate the asymptotic behavior of the solution as $t \longrightarrow \infty$. Let $\lim _{t \rightarrow \infty} u(x, t)=u^{\infty}(x)$, where $u^{\infty}$ is the steady state solution of (3)-(5), satisfying

$$
\begin{aligned}
-\alpha u_{x x}^{\infty}+u_{x}^{\infty}+u^{\infty} u_{x}^{\infty} & =f^{\infty}, \quad t \in(0, \infty), \\
u^{\infty}(x) & =u^{\infty}(x+2 L),
\end{aligned}
$$

where $u_{x x}^{\infty}=\lim _{t \rightarrow \infty} u_{x x}(x, t), u_{x}^{\infty}=$ $\lim _{t \rightarrow \infty} u_{x}(x, t), f^{\infty}(x)=\lim _{t \rightarrow \infty} f(x, t)$.

(A1) Assume the eigenvalue problem,

$$
\begin{aligned}
-\alpha \phi_{x x}+\phi_{x}+u_{x}^{\infty} \phi & =\lambda \phi, \\
\phi(x) & =\phi(x+2 L),
\end{aligned}
$$

has a minimum eigenvalue $\lambda_{0}>0$.

Note that, for any $\phi \in H^{2} \cap H_{p e r}^{1}$, it holds that

$$
\alpha\left\|\phi_{x}\right\|^{2}+\int_{0}^{2 L} u_{x}^{\infty} \phi^{2} d x=\lambda\|\phi\|^{2} \geq \lambda_{0}\|\phi\|^{2} .
$$

Then, we have

$$
\begin{aligned}
& \left(-\alpha \phi_{x x}+\phi_{x}+u^{\infty} \phi_{x}+u_{x}^{\infty} \phi, \phi\right) \\
& =\alpha\left\|\phi_{x}\right\|^{2}+\frac{1}{2} \int_{0}^{2 L} u_{x}^{\infty} \phi^{2} d x, \\
& \geq \frac{\lambda_{0}}{2}\|\phi\|^{2}+\frac{\alpha}{2}\left\|\phi_{x}\right\|^{2} \geq \frac{\alpha}{2}\left\|\phi_{x}\right\|^{2} .
\end{aligned}
$$

Poincaré inequality [16]: For any $\varphi \in H_{p e r}^{2}$, there holds $\left\|\partial_{x}^{k} \varphi\right\| \leq\left(1 / \sqrt{\lambda_{1}}\right)\left\|\partial_{x}^{k+1} \varphi\right\|, \quad k=0,1$, where $\lambda_{1}=\pi^{2} / L^{2}$ is the minimum eigenvalue of the eigenvalue problem: $-\varphi_{x x}=\lambda \varphi$, with $\varphi(x)=\varphi(x+2 L)$.

Let $z=u-u^{\infty}$, from (3)-(5) and (6)-(7), we have

$$
\begin{aligned}
& z_{t}-z_{x x x x t}-\alpha z_{x x}+z_{x}+z z_{x}+u^{\infty} z_{x}+z u_{x}^{\infty}=F \text {, } \\
& t \in(0, \infty) \text {, } \\
& z(x, 0)=u_{0}-u^{\infty}=z_{0}, \\
& z(x, t)=z(x+2 L, t)
\end{aligned}
$$

The weak formulation of (12)-(14) is to find $z(t) \epsilon$ $H_{p e r}^{2}(R)$, such that

$$
\begin{gathered}
\left(z_{t}, v\right)+\left(z_{x x t}, v_{x x}\right)+\alpha\left(z_{x}, v_{x}\right)+\left(z_{x}, v\right)+\left(z z_{x}, v\right) \\
+\left(u^{\infty} z_{x}+z u_{x}^{\infty}, v\right)=(F, v), \quad \forall v \in H_{p e r}^{2} . \\
\left(\text { A2) } u_{0} \in H_{p e r}^{2} \text {, and } \int_{0}^{t}\|F(s)\|^{2} d s \leq M, M>0 .\right.
\end{gathered}
$$

Theorem 1. Under assumptions (A1) and (A2), there holds

$$
\begin{aligned}
\|z\|_{H_{p e r}^{2}}^{2} \leq & \frac{2}{\alpha} \frac{L^{2} \pi^{2}+L^{4}}{\pi^{4}} \int_{0}^{t}\|F(s)\|^{2} d s \\
& +\frac{\pi^{2}+L^{2}}{\pi^{2}}\left\|z_{0}\right\|_{H_{p e r}^{2}}^{2} ;
\end{aligned}
$$

moreover, for given $\epsilon>0$, there exists $T>0$, such that

$$
\|z(t)\|^{2}+\frac{\pi^{2}}{L^{2}}\left\|z_{x}(t)\right\|^{2} \leq \epsilon, \quad \forall t>T .
$$

Proof. Set $v=z$ in (15), and we obtain

$$
\begin{aligned}
& \frac{1}{2} \frac{d}{d t}\left(\|z\|^{2}+\left\|z_{x x}\right\|^{2}\right)+\left(\alpha\left\|z_{x}\right\|^{2}+\left(u^{\infty} z_{x}+u_{x}^{\infty} z, z\right)\right) \\
& \quad=(F, z) .
\end{aligned}
$$

Apply inequality (11), and Young's inequality is to arrive at

$$
\begin{aligned}
& \frac{d}{d t}\left(\|z\|^{2}+\left\|z_{x x}\right\|^{2}\right)+\alpha\left\|z_{x}\right\|^{2} \leq \frac{2}{\sqrt{\lambda_{1}}}\|F\|\left\|z_{x}\right\| \\
& \quad \leq \frac{2}{\alpha \lambda_{1}}\|F\|^{2}+\frac{\alpha}{2}\left\|z_{x}\right\|^{2} .
\end{aligned}
$$

Now, integrating with respect to time from 0 to $t$, we obtain

$$
\begin{aligned}
& \|z\|^{2}+\left\|z_{x x}\right\|^{2}+\frac{\alpha}{2} \int_{0}^{t}\left\|z_{x}(s)\right\|^{2} d s \\
& \quad \leq \frac{2}{\alpha \lambda_{1}} \int_{0}^{t}\|F(s)\|^{2} d s+\left\|z_{0}\right\|^{2}+\left\|z_{0 x x}\right\|^{2} .
\end{aligned}
$$

Applying the Poincaré inequalities, we obtain

$$
\begin{aligned}
\|z\|^{2}+\left\|z_{x x}\right\|^{2}= & \|z\|^{2}+\frac{L^{2}}{\pi^{2}+L^{2}}\left\|z_{x x}\right\|^{2} \\
& +\frac{\pi^{2}}{\pi^{2}+L^{2}}\left\|z_{x x}\right\|^{2} \\
\geq & \|z\|^{2}+\frac{L^{2}}{\pi^{2}+L^{2}} \frac{\pi^{2}}{L^{2}}\left\|z_{x}\right\|^{2} \\
& +\frac{\pi^{2}}{\pi^{2}+L^{2}}\left\|z_{x x}\right\|^{2} \geq \frac{\pi^{2}}{\pi^{2}+L^{2}}\|z\|_{H_{p e r}^{2}}^{2} .
\end{aligned}
$$

Substiuting (21) into (20), we have

$$
\begin{aligned}
\|z\|_{H_{p e r}^{2}}^{2} \leq & \frac{2}{\alpha} \frac{L^{2} \pi^{2}+L^{4}}{\pi^{4}} \int_{0}^{t}\|F(s)\|^{2} d s \\
& +\frac{\pi^{2}+L^{2}}{\pi^{2}}\left\|z_{0}\right\|_{H_{p e r}^{2}}^{2} .
\end{aligned}
$$

where $F=f-f^{\infty}$. 
On the other hand, using Poincaré inequality again, we have

$$
\begin{aligned}
& \frac{\alpha}{2} \int_{0}^{t}\left\|z_{x}(s)\right\|^{2} d s \\
& \quad=\frac{\alpha}{2} \int_{0}^{t} \frac{\pi^{4}}{\pi^{4}+L^{4}}\left\|z_{x}(s)\right\|^{2}+\frac{L^{4}}{\pi^{4}+L^{4}}\left\|z_{x}(s)\right\|^{2} d s \\
& \quad \geq \frac{\alpha}{2} \int_{0}^{t} \frac{\pi^{4}}{\pi^{4}+L^{4}}\left\|z_{x}(s)\right\|^{2}+\frac{L^{2} \pi^{2}}{\pi^{4}+L^{4}}\|z(s)\|^{2} d s \\
& \quad=\gamma \int_{0}^{t}\left(\|z(s)\|^{2}+\frac{\pi^{2}}{L^{2}}\left\|z_{x}(s)\right\|^{2}\right) d s,
\end{aligned}
$$

where $\gamma=\alpha \pi^{2} L^{2} / 2\left(\pi^{4}+L^{4}\right)$. Substituting (23) into (20) yields

$$
\begin{aligned}
& \|z(t)\|^{2}+\frac{\pi^{2}}{L^{2}}\left\|z_{x}(t)\right\|^{2} \\
& \quad+\gamma \int_{0}^{t}\left(\|z(s)\|^{2}+\frac{\pi^{2}}{L^{2}}\left\|z_{x}(s)\right\|^{2}\right) d s \\
& \leq \frac{2}{\alpha \lambda_{1}} \int_{0}^{t}\|F(s)\|^{2} d s+\left\|z_{0}\right\|_{H_{p e r}^{2}}^{2} .
\end{aligned}
$$

That is,

$$
\lim _{t \rightarrow \infty}\left(\|z(t)\|^{2}+\frac{\pi^{2}}{L^{2}}\left\|z_{x}(t)\right\|^{2}\right)=0 .
$$

Hence, we finish the proof of (17).

\section{Time-Discrete and Error Analysis}

In this section, we present the semidiscrete scheme for the solution of (3)-(5). First, we introduce a Crank-Nicholson method to discrete time. Let $K$ be a positive integer, $\Delta t=T / K$ is the time step, and $t_{n}=n \Delta t, n=0,1, \ldots, K-1$ are the mesh points.

Consider the following time-discrete scheme:

$$
\begin{aligned}
& \frac{u^{n+1}-u^{n}}{\Delta t}+\partial_{x} u^{n+1 / 2}-\frac{1}{\Delta t}\left(\partial_{x}^{4} u^{n+1}-\partial_{x}^{4} u^{n}\right) \\
& +\frac{1}{6}\left(2 \partial_{x} u^{n+1 / 2}\left(3 u^{n}-u^{n-1}\right)\right. \\
& \left.+u^{n+1 / 2} \partial_{x}\left(3 u^{n}-u^{n-1}\right)\right)-\alpha \partial_{x}^{2} u^{n+1 / 2}=0,
\end{aligned}
$$

$n \geq 1$.

We have the following stability result.

Theorem 2. The semidiscrete scheme (26) is unconditionally stable, such that

$$
E\left(u^{n+1}\right) \leq E\left(u^{0}\right), \quad n=0,1, \ldots, K-1,
$$

where

$$
E\left(u^{n}\right)=\left\|u^{n}\right\|^{2}+\left\|\partial_{x}^{2} u^{n}\right\|^{2}
$$

Proof. Taking the inner product of (26) with $2 \Delta t u^{n+(1 / 2)}$, we get

$$
\begin{gathered}
\left\|u^{n+1}\right\|_{0}^{2}-\left\|u^{n}\right\|^{2}+\left\|\partial_{x}^{2} u^{n+1}\right\|^{2}-\left\|\partial_{x}^{2} u^{n}\right\|^{2} \\
+2 \Delta t \alpha\left\|\partial_{x} u^{n+1 / 2}\right\|^{2}=0 .
\end{gathered}
$$

That is,

$$
\left\|u^{n+1}\right\|^{2}+\left\|\partial_{x}^{2} u^{n+1}\right\|^{2} \leq\left\|u^{n}\right\|^{2}+\left\|\partial_{x}^{2} u^{n}\right\|^{2} .
$$

This concludes the proof.

We will consider Fourier-Galerkin spectral method for the discretization equations (27). We will present some error estimate for full-discretization schemes. First, let us define

$$
S_{N}=\left\{u \mid u(x)=\sum_{|k| \leq N / 2} \widehat{u}_{k} e_{k}, e_{k}=e^{-i k x}\right\} .
$$

Denote $\pi_{N}: L^{2} \longrightarrow S_{N}$ to be the $L^{2}$-projection operator which satisfies

$$
\left(\pi_{N} \phi-\phi, \psi\right)=0, \quad \forall \psi \in S_{N} .
$$

We also define the $H^{2}$-projection operator $\pi_{N}^{1}: H^{2} \longrightarrow S_{N}$ by

$$
\left(\partial_{x}^{2}\left(\pi_{N}^{1} \phi-\phi\right), \partial_{x}^{2} \psi\right)=0, \quad \forall \psi \in S_{N}
$$

We have the following estimate [17]:

$$
\left\|\phi-\pi_{N} \phi\right\|_{s} \leq c N^{s-m}\|\phi\|_{m}, \quad \forall \phi \in H^{m}, m \geq 0 .
$$

Consider the full-discretization Fourier-Galerkin spectral method to (26) as follows: find $u_{N}^{n+1} \in S_{N}$, such that

$$
\begin{aligned}
& \frac{1}{\Delta t}\left(u_{N}^{n+1}-u_{N}^{n}, \psi_{N}\right)+\left(\partial_{x} u_{N}^{n+1 / 2}, \psi_{N}\right)-\frac{1}{\Delta t}\left(\partial_{x}^{4} u_{N}^{n+1}\right. \\
& \left.\quad-\partial_{x}^{4} u_{N}^{n}, \psi_{N}\right)+\frac{1}{6}\left(2 \partial_{x} u_{N}^{n+1 / 2}\left(3 u_{N}^{n}-u_{N}^{n-1}\right)\right. \\
& \left.\quad+u_{N}^{n+1 / 2} \partial_{x}\left(3 u_{N}^{n}-u_{N}^{n-1}\right), \psi_{N}\right)-\alpha\left(\partial_{x}^{2} u_{N}^{n+1 / 2}, \psi_{N}\right) \\
& \quad=0, \quad n \geq 1, \quad \psi_{N} \in S_{N} .
\end{aligned}
$$

Theorem 3. Let $\left\{u_{N}^{n}\right\}_{n=1}^{M-1}$ be the solutions of (26), and we derive that

$$
\left\|u_{N}^{n+1}\right\|^{2}+\left\|\partial_{x}^{2} u_{N}^{n+1}\right\|^{2} \leq\left\|u_{N}^{0}\right\|^{2}+\left\|\partial_{x}^{2} u_{N}^{0}\right\|^{2}
$$

We denote the truncation error $r^{n+1 / 2}(x)=r_{1}^{n+1 / 2}(x)+$ $r_{2}^{n+1 / 2}(x)$; here,

$$
\begin{aligned}
r_{1}^{n+1 / 2}(x):= & \frac{u\left(x, t_{n+1}\right)-u\left(\cdot, t_{n}\right)}{\Delta t}-\partial_{t} u\left(x, t_{n+1 / 2}\right), \\
r_{2}^{n+1 / 2}(x):= & \frac{\partial_{x}^{4} u\left(x, t_{n+1}\right)-\partial_{x}^{4} u\left(\cdot, t_{n}\right)}{\Delta t} \\
& -\partial_{x}^{2} \partial_{t} u\left(x, t_{n+1 / 2}\right) .
\end{aligned}
$$


From Taylor expansion, we have

$$
\left\|r^{n+1 / 2}\right\|_{0}^{2} \leq c \Delta t^{4}
$$

We also define the following error functions:

$$
\begin{aligned}
& \tilde{e}^{n}=\pi_{N} u\left(t_{n}\right)-u_{N}^{n}, \\
& \hat{e}^{n}=u\left(t_{n}\right)-\pi_{N} u\left(t_{n}\right), \\
& e^{n}=\hat{e}^{n}+\widetilde{e}^{n} .
\end{aligned}
$$

We show the error estimate of the full-discretization problem (35) in the following theorem.

Theorem 4. Suppose $u(x, t)$ is the exact solution of (3)-(5) and $\left\{u_{N}^{n}\right\}_{n=1}^{M-1}$ are the solutions of (38); then, we have

$$
E\left(u\left(\cdot, t_{n}\right)-u_{N}^{n}\right) \leq c\left(\Delta t^{2}+N^{2-m}\right) .
$$

Proof. Subtracting (35) from a reformulation of (3) at $t_{n+1 / 2}$, we obtain

$$
\begin{aligned}
& \left(\frac{\widetilde{e}_{N}^{n+1}-\widetilde{e}_{N}^{n}}{\Delta t}, \psi_{N}\right)+\left(\partial_{x} \tilde{e}_{N}^{n+1 / 2}, \psi_{N}\right)+\frac{1}{\Delta t}\left(\partial_{x}^{2} \widetilde{e}_{N}^{n+1}\right. \\
& \left.-\partial_{x}^{2} \widetilde{e}_{N}^{n}, \partial_{x} \psi_{N}\right)+\alpha\left(\partial_{x} \widetilde{e}_{N}^{n+1 / 2}, \partial_{x} \psi_{N}\right)+\left(u\left(t_{n+1 / 2}\right)\right. \\
& \cdot \partial_{x} u\left(t_{n+1 / 2}\right)-\frac{1}{6}\left(2 \partial_{x} u_{N}^{n+1 / 2}\left(3 u_{N}^{n}-u_{N}^{n-1}\right)\right. \\
& \left.\left.+u_{N}^{n+1 / 2} \partial_{x}\left(3 u_{N}^{n}-u_{N}^{n-1}\right)\right), \psi_{N}\right)=\left(r^{n+1 / 2}, \psi_{N}\right) \\
& +\left(\left(\pi_{N}^{1}-I\right) \partial_{x} u\left(\cdot, t_{n+1 / 2}\right), \psi_{N}\right)+\frac{1}{\Delta t}\left(\left(\pi_{N}^{1}-I\right)\right. \\
& \left.\cdot\left(u\left(\cdot, t_{n+1}\right)-u\left(\cdot, t_{n}\right)\right), \psi_{N}\right)+\frac{1}{\Delta t}\left(\left(\pi_{N}^{1}-I\right)\right. \\
& \left.\cdot\left(\partial_{x}^{2} u\left(\cdot, t_{n+1}\right)-\partial_{x}^{2} u\left(\cdot, t_{n}\right)\right), \psi_{N}\right), \quad \psi_{N} \in S_{N} .
\end{aligned}
$$

$$
\text { Let } \psi_{N}=2 \Delta t \widetilde{e}_{N}^{n+1 / 2} \text {, and }
$$

$$
\begin{aligned}
& E\left(\tilde{e}^{n+1}\right)-E\left(\tilde{e}^{n}\right) \leq 2 \Delta t\left\|r^{n+1 / 2}\right\|\left\|\tilde{e}_{N}^{n+1 / 2}\right\|+2 \Delta t \|\left(\pi_{N}^{1}\right. \\
& \quad-I) \partial_{x} u\left(\cdot, t_{n+1}\right)\|\| \tilde{e}_{N}^{n+1 / 2}\|+2\|\left(\pi_{N}^{1}-I\right)\left(u\left(\cdot, t_{n+1}\right)\right. \\
& \left.\quad-u\left(\cdot, t_{n}\right)\right)\|\| \tilde{e}_{N}^{n+1 / 2}\|+2\|\left(\pi_{N}^{1}-I\right)\left(\partial_{x} u\left(\cdot, t_{n+1}\right)\right. \\
& \left.\quad-\partial_{x} u\left(\cdot, t_{n}\right)\right)\|\| \partial_{x} \tilde{e}_{N}^{n+1 / 2}\|+2 \Delta t\| u\left(\cdot, t_{n+1 / 2}\right) \\
& \quad \cdot \partial_{x} u\left(\cdot, t_{n+1 / 2}\right)-\frac{1}{6}\left(2 \partial_{x} u_{N}^{n+1}\left(3 u_{N}^{n}-u_{N}^{n-1}\right)\right. \\
& \left.\quad+u_{N}^{n+1} \partial_{x}\left(3 u_{N}^{n}-u_{N}^{n-1}\right)\right)\|\| \tilde{e}_{N}^{n+1} \|=: Q_{1}+Q_{2}+Q_{3} \\
& \quad+Q_{4} .
\end{aligned}
$$

Using Cauchy-Schwarz and Young's inequality, we have

$$
\begin{aligned}
Q_{1} \leq & \Delta t\left\|r^{n+1 / 2}\right\|^{2}+\Delta t\left\|\left(\pi_{N}^{1}-I\right) \partial_{x} u\left(\cdot, t_{n+1 / 2}\right)\right\|^{2} \\
& +2 \Delta t\left\|\widetilde{e}_{N}^{n+1 / 2}\right\|^{2}, \\
Q_{2} \leq & \int_{t_{n}}^{t_{n+1}}\left\|\left(\pi_{N}^{1}-I\right) \partial_{t} u(\cdot, t)\right\|^{2} d t+\Delta t\left\|\widetilde{e}_{N}^{n+1 / 2}\right\|^{2}, \\
Q_{3} \leq & \int_{t_{n}}^{t_{n+1}}\left\|\left(\pi_{N}^{1}-I\right) \partial_{x}^{2} \partial_{t} u(\cdot, t)\right\|^{2}+\Delta t\left\|\partial_{x}^{2} \widetilde{e}_{N}^{n+1 / 2}\right\|^{2}, \\
Q_{4} \leq & \Delta t\left(\left\|D_{1}\right\|^{2}+\left\|D_{2}\right\|^{2}+\left\|\widetilde{e}_{N}^{n+1 / 2}\right\|^{2}\right),
\end{aligned}
$$

where

$$
\begin{aligned}
& D_{1}=\frac{2}{3} u\left(\cdot, t_{n+1 / 2}\right) \partial_{x} u\left(\cdot, t_{n+1 / 2}\right) \\
& -\frac{1}{3}\left(3 u_{N}^{n}-u_{N}^{n-1}\right) \partial_{x} u_{N}^{n+1 / 2} \\
& =\frac{2}{3} u\left(\cdot, t_{n+1 / 2}\right) \partial_{x} u\left(\cdot, t_{n+1 / 2}\right) \\
& -\frac{1}{3}\left(3 u\left(\cdot, t_{n}\right)-u\left(\cdot, t_{n-1}\right)\right) \partial_{x} u\left(\cdot, t_{n+1 / 2}\right) \\
& +\frac{1}{3}\left(3 u\left(\cdot, t_{n}\right)-u\left(\cdot, t_{n-1}\right)\right) \partial_{x} u\left(\cdot, t_{n+1 / 2}\right) \\
& -\frac{1}{3}\left(3 u_{N}^{n}-u_{N}^{n-1}\right) \partial_{x} u\left(\cdot, t_{n+1 / 2}\right) \\
& +\frac{1}{3}\left(3 u_{N}^{n}-u_{N}^{n-1}\right) \partial_{x} u\left(\cdot, t_{n+1 / 2}\right) \\
& -\frac{1}{3}\left(3 u_{N}^{n}-u_{N}^{n-1}\right) \partial_{x} u_{N}^{n+1 / 2} \\
& D_{2}=\frac{1}{3} u\left(t_{n+1 / 2}\right) \partial_{x} u\left(t_{n+1}\right) \\
& -\frac{1}{3} u_{N}^{n+1 / 2} \partial_{x}\left(3 u_{N}^{n}-u_{N}^{n-1}\right) \\
& =\frac{1}{3} u\left(t_{n+1 / 2}\right) \partial_{x} u\left(\cdot, t_{n+1}\right) \\
& -\frac{1}{3} u\left(\cdot, t_{n+1 / 2}\right) \partial_{x}\left(3 u\left(\cdot, t_{n}\right)-u\left(\cdot, t_{n-1}\right)\right) \\
& +\frac{1}{3} u\left(\cdot, t_{n+1 / 2}\right) \partial_{x}\left(3 u\left(\cdot, t_{n}\right)-u\left(\cdot, t_{n-1}\right)\right) \\
& -\frac{1}{3} u_{N}^{n+1 / 2} \partial_{x}\left(3 u\left(\cdot, t_{n}\right)-u\left(\cdot, t_{n-1}\right)\right) \\
& +\frac{1}{3} u_{N}^{n+1 / 2} \partial_{x}\left(3 u\left(\cdot, t_{n}\right)-u\left(\cdot, t_{n-1}\right)\right) \\
& -\frac{1}{3} u_{N}^{n+1 / 2} \partial_{x}\left(3 u_{N}^{n}-u_{N}^{n-1}\right) .
\end{aligned}
$$


Applying Taylor expansion and Young's inequality,

$$
\begin{aligned}
\frac{1}{3}\left\|D_{1}\right\|_{0}^{2} \leq & \Delta t^{3}\left\|\partial_{x} u\left(\cdot, t_{n+1 / 2}\right)\right\|_{\infty}^{2} \int_{t_{n}}^{t_{n+1}}\left\|\partial_{t}^{2} u(\cdot, t)\right\|_{0}^{2} d t \\
& +\left\|\partial_{x} u\left(\cdot, t_{n+1 / 2}\right)\right\|_{\infty}^{2}\left\|3 e_{N}^{n}-e_{N}^{n-1}\right\|^{2} \\
& +\left\|3 u_{N}^{n}-u_{N}^{n-1}\right\|_{\infty}^{2}\left\|\partial_{x} e_{N}^{n+1 / 2}\right\|^{2}, \\
\leq & c\left(\Delta t^{4}+\left\|3 e_{N}^{n}-e_{N}^{n-1}\right\|^{2}+\left\|\partial_{x} e_{N}^{n+1 / 2}\right\|^{2}\right) . \\
\frac{1}{3}\left\|D_{2}\right\|_{0}^{2} \leq & \Delta t^{3}\left\|u\left(\cdot, t_{n+1 / 2}\right)\right\|_{\infty}^{2} \int_{t_{n}}^{t_{n+1}}\left\|\partial_{x} \partial_{t}^{2} u(\cdot, t)\right\|^{2} d t \\
& +\left\|\partial_{x}\left(3 u\left(\cdot, t_{n}\right)-u\left(\cdot, t_{n-1}\right)\right)\right\|_{\infty}^{2}\left\|e_{N}^{n+1 / 2}\right\|^{2} \\
& +\left\|u_{N}^{n+1 / 2}\right\|_{\infty}^{2}\left\|\partial_{x}\left(3 e_{N}^{n}-e_{N}^{n-1}\right)\right\|_{0}^{2} \\
\leq & c\left(\Delta t^{4}+\left\|\partial_{x}\left(3 e_{N}^{n}-e_{N}^{n-1}\right)\right\|^{2}+\left\|e_{N}^{n+1 / 2}\right\|^{2}\right) .
\end{aligned}
$$

Then, we obtain

$$
\begin{aligned}
& E\left(\tilde{e}^{n+1}\right)-E\left(\widetilde{e}^{n}\right) \leq \Delta t\left\|r^{n+1 / 2}\right\|^{2} \\
& +\Delta t\left\|\left(\pi_{N}^{1}-I\right) \partial_{x} u\left(\cdot, t_{n+1}\right)\right\|^{2}+2 \Delta t\left\|e_{N}^{n+1}\right\|^{2} \\
& \quad+\int_{t_{n}}^{t_{n+1}}\left\|\left(\pi_{N}^{1}-I\right) \partial_{t} u(\cdot, t)\right\|^{2} d t+\Delta t\left\|e_{N}^{n+1}\right\|^{2}, \\
& +\int_{t_{n}}^{t_{n+1}}\left\|\left(\pi_{N}^{1}-I\right) \partial_{x} \partial_{t} u(\cdot, t)\right\|^{2} d t+\Delta t\left\|\partial_{x} \widetilde{e}_{N}^{n+1}\right\|^{2}, \\
& +c \Delta t\left(\left\|\partial_{x}\left(3 e_{N}^{n}-e_{N}^{n-1}\right)\right\|^{2}+\left\|3 e_{N}^{n}-e_{N}^{n-1}\right\|^{2}\right. \\
& \left.\quad+\left\|e_{N}^{n+1 / 2}\right\|^{2}+\left\|\partial_{x} e_{N}^{n+1 / 2}\right\|^{2}\right) .
\end{aligned}
$$

Adding up for $n=1, \ldots, k$ and using the following inequality,

$$
\left\|\partial_{x} u_{N}^{n}\right\|^{2} \leq c\left(\left\|u_{N}^{n}\right\|^{2}+\left\|\partial_{x}^{2} u_{N}^{n}\right\|^{2}\right)
$$

we have

$$
E\left(\widetilde{e}^{n+1}\right) \leq E\left(\widetilde{e}^{1}\right)+c\left(\Delta t^{4}+N^{4-2 m}\right)+c \Delta t \sum_{n=1}^{k} E\left(\widetilde{e}^{n}\right)
$$

For the first step, it is to verify that

$$
E\left(\tilde{e}^{1}\right) \leq c\left(\Delta t^{4}+N^{4-2 m}\right) .
$$

By using Gronwall inequality, we have

$$
E\left(\widetilde{e}^{n+1}\right) \leq c\left(\Delta t^{4}+N^{4-2 m}\right) .
$$

Therefore, estimate (42) is proved.

\section{Numerical Results}

This section presents several numerical examples to confirm the accuracy and applicability of schemes (35) for solving Rosenau-Burgers equation. Fisrt, we let

$$
u_{N}^{n}=\sum_{k=-N / 2}^{N / 2-1} \widehat{u}_{k}^{n+1} \exp \left(-\frac{i \pi k x}{L}\right)
$$

And we obtain the following linear system:

$$
\begin{aligned}
& \frac{1}{\Delta t}\left(\widehat{u}_{k}^{n+1}-\widehat{u}_{k}^{n}\right)\left(1+\left(\frac{\pi k}{L}\right)^{4}\right)+\left(\frac{i \pi k}{L}+\alpha\left(\frac{\pi k}{L}\right)^{2}\right) \\
& \cdot \widehat{u}_{k}^{n+1 / 2}+\frac{1}{6}\left\{2\left(3 u_{N}^{n}-u_{N}^{n-1}\right) \partial_{x} u_{N}^{n+1 / 2}\right. \\
& \left.+\partial_{x}\left(3 u_{N}^{n}-u_{N}^{n-1}\right) u_{N}^{n+(1 / 2)}\right\}_{k}=0,
\end{aligned}
$$

where $\widehat{u}_{k}$ or $\{u\}_{k}$ represents the $k$ th mode Fourier coefficient of the function $u$.

4.1. Verification of the Temporal Convergence Order. We use the following quantity to compute the convergence rate in time direction [18]:

$$
p=\log _{2}\left(\frac{\left\|u_{N}^{n, 2 \Delta t}-u_{N}^{2 n, \Delta t}\right\|_{0}}{\left\|u_{N}^{2 n, \Delta t}-u_{N}^{4 n, \Delta t / 2}\right\|_{0}}\right)
$$

The full discrete problem (52) is solved in $\Omega=(0,2 \pi)$ with $T=1$ and $N=60$. Tables $1-3$ display the temporal convergence orders for different values of $\alpha$ and initial condition $u_{0}$. As shown in Tables 1-3, our numerical scheme (52) is of the 2 nd order accuracy in time, which is confirmed with the theoretical result in Theorem 4.

4.2. Asymptotic Properties of Solutions. In order to verify the asymptotic behavior of $z(x, t)$, the effect of $\alpha$ and initial condition $u_{0}$ will be investigated. Let $\alpha=1$ and $f=$ $\exp (-t) \cos x-\exp (-t) \sin x+\exp (-2 t) \cos x \sin x$; then, the exact solution is $u(x, t)=\exp (-t) \sin x$, and $u^{\infty}=f^{\infty}=0$. The numerical result in Figure 1 shows the convergence of the solution $u(x, t)$ to its steady-state solution $u^{\infty}$. When $\alpha=1$, and $f=(\exp (-t)+1) \cos x-2 \exp (-t) \cos x-$ $\sin x(\exp (-t)+1)-\cos x \sin x(\exp (-t)+1)^{2}$ in (3), the exaction solution is $u=(\exp (-t)+1) \cos x$, the steady-state solution is $u^{\infty}=\cos x$, and $f^{\infty}=\cos x-\sin x-\sin x \cos x$. It is easy to verify that both $u^{\infty}$ and $F$ satisfy the assumptions of (A1) and (A2). Figure 2 indicates that the numerical solution 
TABLE 1: Temporal convergence orders for $u_{0}=\cos x$.

\begin{tabular}{lcccc}
\hline$\alpha \backslash \Delta t$ & $\Delta t=1.00 \mathrm{E}-1$ & $\Delta t=5.00 \mathrm{E}-2$ & $\Delta t=1.00 \mathrm{E}-2$ & $\Delta t=5.00 \mathrm{E}-3$ \\
\hline$\alpha=0$ & 1.9933 & 2.0010 & 2.0009 & 2.0005 \\
$\alpha=1$ & 1.9428 & 1.9712 & 1.9942 & 1.9972 \\
$\alpha=3$ & 1.8833 & 1.9262 & 1.9825 & 1.9910 \\
$\alpha=5$ & 1.7717 & 1.8503 & 1.9629 & 1.9810 \\
\hline
\end{tabular}

TABLE 2: Temporal convergence orders for $u_{0}=\sin x+\cos x$.

\begin{tabular}{lcccc}
\hline$\alpha \backslash \Delta t$ & $\Delta t=1.00 \mathrm{E}-1$ & $\Delta t=5.00 \mathrm{E}-2$ & $\Delta t=1.00 \mathrm{E}-2$ & $\Delta t=5.00 \mathrm{E}-3$ \\
\hline$\alpha=0$ & 1.9870 & 1.9992 & 2.0007 & 2.0005 \\
$\alpha=1$ & 1.9329 & 1.9667 & 1.9934 & 1.9968 \\
$\alpha=3$ & 1.8715 & 1.9187 & 1.9806 & 1.9901 \\
$\alpha=5$ & 1.7528 & 1.8371 & 1.9593 & 1.9791 \\
\hline
\end{tabular}

TABLE 3: Temporal convergence orders for $u_{0}=\sin 3 x \cos 2 x$.

\begin{tabular}{lcccc}
\hline$\alpha \backslash \Delta t$ & $\Delta t=1.00 \mathrm{E}-1$ & $\Delta t=5.00 \mathrm{E}-2$ & $\Delta t=1.00 \mathrm{E}-2$ & $\Delta t=5.00 \mathrm{E}-3$ \\
\hline$\alpha=0$ & 1.9959 & 2.0008 & 2.0006 & 2.0004 \\
$\alpha=1$ & 1.9542 & 1.9767 & 1.9953 & 1.9978 \\
$\alpha=3$ & 1.8928 & 1.9325 & 1.9840 & 1.9918 \\
$\alpha=5$ & 1.7906 & 1.8635 & 1.9664 & 1.9828 \\
\hline
\end{tabular}

of $u(x, t)$ converges to $u^{\infty}$ as $t \longrightarrow \infty$. In Figure 3 , we plot the numerical solution at $\alpha=0$, we can observe that $u(x, t)$ oscillates around 0 . In Figures $4-5$, we observed that the numerical solutions decay almost to zero when $\alpha$ becomes larger. This implies that the impact of $\alpha$ is significant in the long time behavior. The figures are for $z(x, t)$ indicated in the result discussions.

The reference solutions were obtained by using the numerical scheme (35) with $N=128$ and $\delta t=5 \times 10^{-5}$. We present $L^{2}$ and $H^{1}$ errors for $u$. The results are summarized in Figure 6, in which we conclude that the full-discrete scheme (35) reaches the second-order convergence rate in time direction. In order to test the spatial spectral accuracy, error functions for $N$ are given in Figure 7, which show that our numerical method has good convergence behavior. We observe it reaches spectral convergence in space, if the error in spatial direction is negligible.

\section{Conclusion}

This article studies the asymptotic stability of RosenauBurgers equation. Under suitable assumptions on an eigenvalue problem and the forcing function, the convergence order of the Rosenau-Burgers equation to its steady-state problem is derived. Then, we propose a linearized numerical method for solving this nonlinear Rosenau-Burgers equation. The numerical method is combined with a finite difference scheme in time and Fourier-spectral method in space. We have derived a discrete stability inequality and error estimate for the numerical scheme which leads to 2 nd-order accuracy in time and spectral accuracy in space. Numerical examples are illustrated to verify the theoretical results.

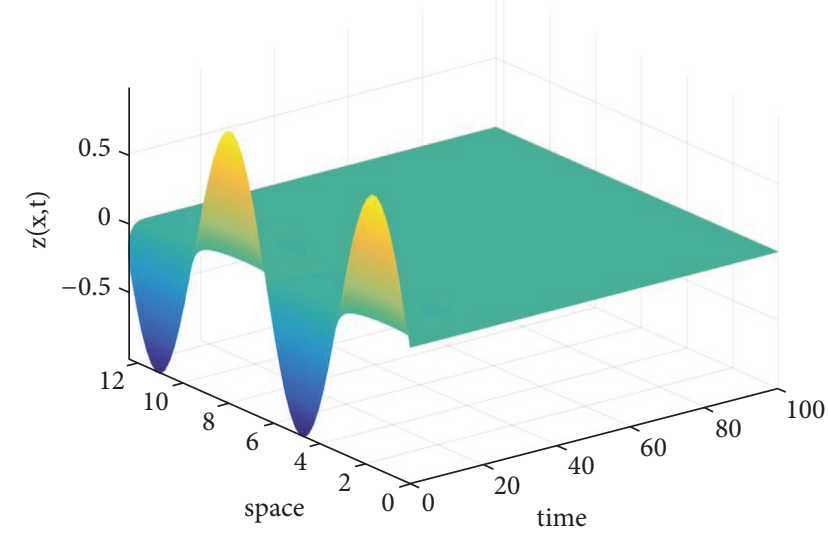

FIGURE 1: Approximation solutions of $z(x, t)$ at $\alpha=1$ and $u_{0}=\sin x$.

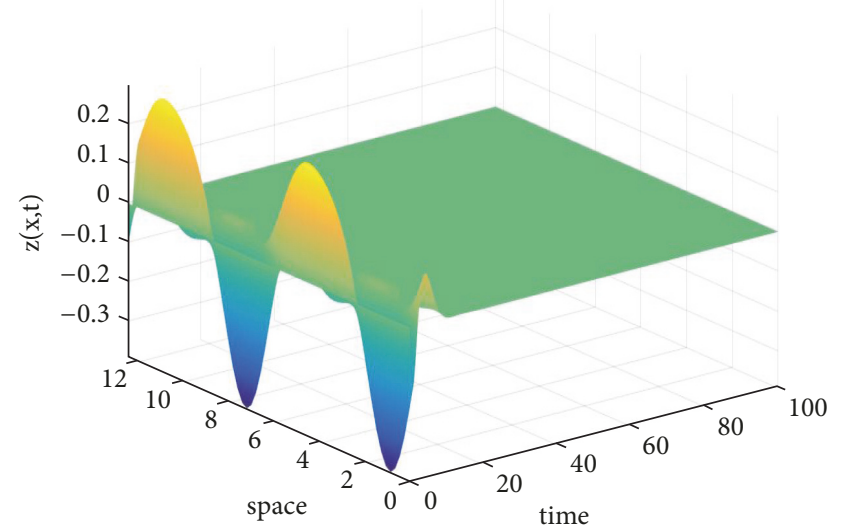

FIgURE 2: Approximation solutions of $z(x, t)$ at $\alpha=1$ and $u_{0}=$ $\cos x$. 


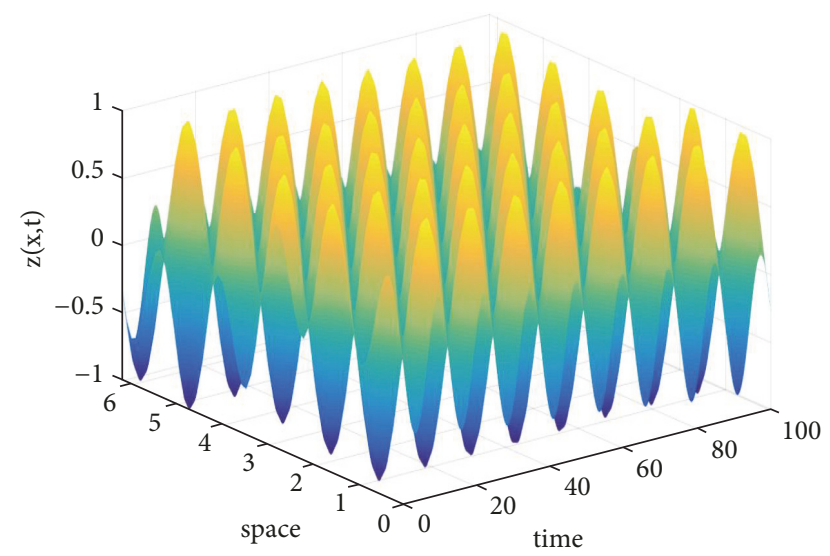

FIgURE 3: The graph of approximation solutions at $\alpha=0, \Delta t=$ $0.01, N=60, u_{0}=\sin 3 x \cos 2 x$.

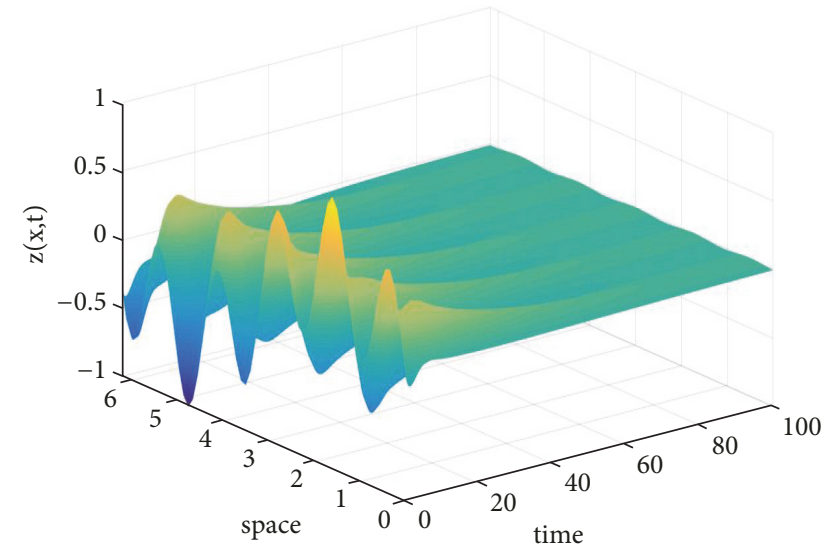

FigURE 4: The graph of approximation solutions at $\alpha=1, \Delta t=$ $0.01, N=60, u_{0}=\sin 3 x \cos 2 x$.

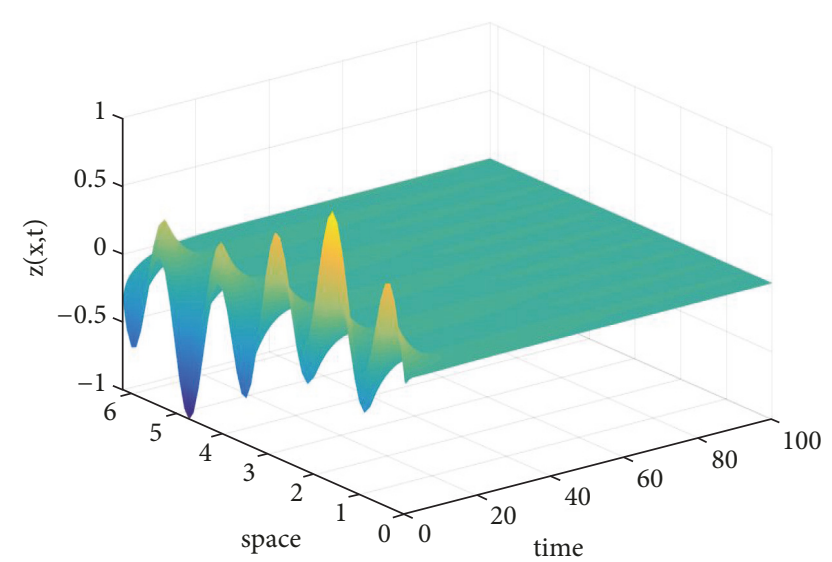

FIgURE 5: The graph of approximation solution at $\alpha=5, \Delta t=$ $0.01, N=60, u_{0}=\sin 3 x \cos 2 x$.

\section{Data Availability}

The data used to support the findings of this study are available from the corresponding author upon request.

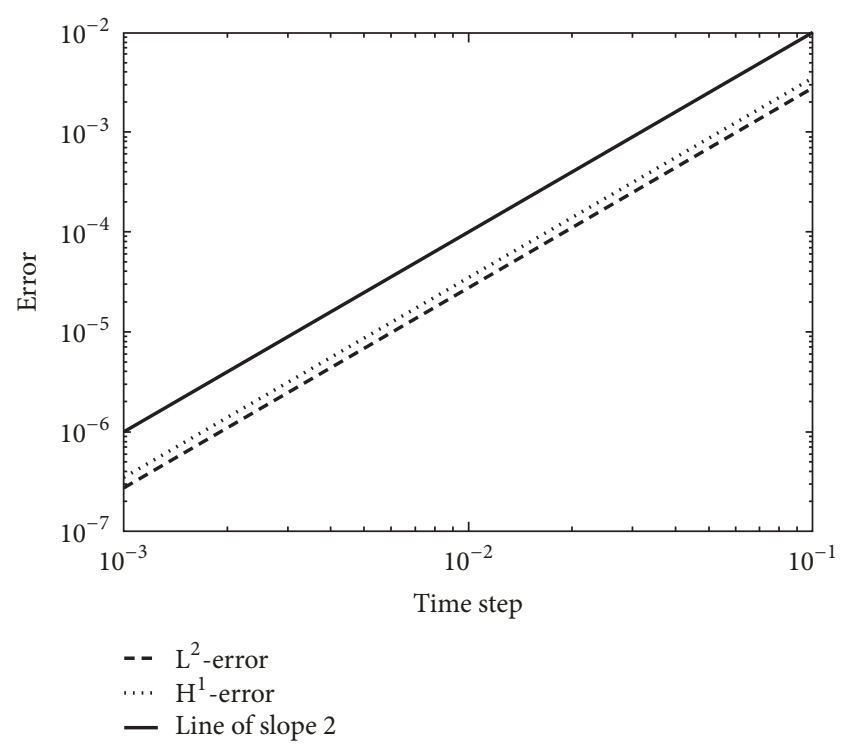

Figure 6: Errors in time direction with $u_{0}=\sin 3 x \cos 2 x$.

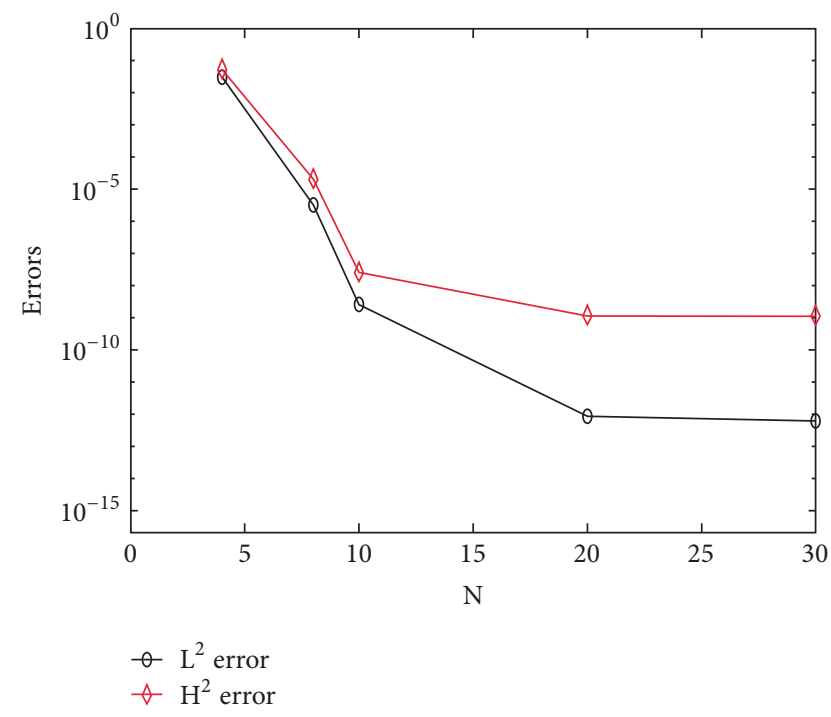

FIGURE 7: Spatial $L^{2}$ and $H^{2}$ errors in space direction with $u_{0}=$ $\sin 3 x \cos 20 x$.

\section{Conflicts of Interest}

The authors declare that they have no conflicts of interest.

\section{Acknowledgments}

The work of Jun Zhang is supported by the Chinese Postdoc Foundation Grant (no. 2019M653490) and the academic project of Guizhou University of Finance and Economics (no. [2018]5774-033). The work of Zixin Liu is supported by National Natural Science Foundation of China (no. 61472093), Guizhou Province University science and technology top talents project (no. KY[2018]047), and Guizhou University of Finance and Economics (no. 2018XZD01). This work of Fubiao Lin is supported by the Science 
Technology Foundation of Guizhou Education Department (no. QJK[KY] [2019]1051) and academic project of Guizhou University of Finance and Economics (no. [2017] 5736-020). The work of Jianjun Jiao is supported by National Natural Science Foundation of China (nos. 11791019 and 11361014), the Joint Fund Project of Department of Commerce with GUFE (no. 2016SWBZD18), the Science Technology Foundation of Guizhou Education Department (nos. QJK[KY]2018019, [2019]1051), the Project of High Level Creative Talents in Guizhou Province (no. 20164035), and the Guizhou Province University science and technology top talents project (no. 2018-047).

\section{References}

[1] P. Rosenau, "A quasi-continuous description of a nonlinear transmission line," Physica Scripta, vol. 34, no. 6B, pp. 827-829, 1986.

[2] P. Rosenau, "Dynamics of dense discrete systems," Progress of Theoretical and Experimental Physics, vol. 79, no. 5, pp. 1028$1042,1988$.

[3] M. A. Park, "On the Rosenau equation in multidimensional space," Nonlinear Analysis: Theory, Methods \& Applications, vol. 21, no. 1, pp. 77-85, 1993.

[4] M. Mei, "Long-time behavior of solution for Rosenau-Burgers equation (i)," Applicable Analysis: An International Journal, vol. 63, no. 3-4, pp. 315-330, 1996.

[5] M. Mei, "Long-time behavior of solution for Rosenau-Burgers equation (ii)," Applicable Analysis: An International Journal, vol. 68, no. 3-4, pp. 333-356, 1998.

[6] L. Liu and M. Mei, "A better asymptotic profile of RosenauBurgers equation," Applied Mathematics and Computation, vol. 131, no. 1, pp. 147-170, 2002.

[7] L. Liu, M. Mei, and Y. S. Wong, "Asymptotic behavior of solutions to the Rosenau-Burgers equation with a periodic initial boundary," Nonlinear Analysis Theory Methods and Applicationsl, vol. 67, no. 8, pp. 2527-2539, 2007.

[8] S.-i. Kinami, M. Mei, and S. Omata, "Convergence to diffusion waves of the solutions for Benjamin-Bona-Mahony-Burgers equations," Applicable Analysis: An International Journal, vol. 75, no. 3-4, pp. 317-340, 2000.

[9] M. Mei and C. Schmeiser, "Asymptotic profiles of solutions for the BBM-Burgers equation," Funkcialaj Ekvacioj. Serio Internacia, vol. 44, no. 1, pp. 151-170, 2001.

[10] S. K. Chung and S. N. Ha, "Finite element Galerkin solutions for the Rosenau equation," Applicable Analysis: An International Journal, vol. 54, no. 1-2, pp. 39-56, 1994.

[11] S. K. Chung and A. K. Pani, "Numerical methods for the Rosenau equation," Applicable Analysis: An International Journal, vol. 77, no. 3-4, pp. 351-369, 2001.

[12] S. K. Chung, "Finite difference approximate solutions for the Rosenau equation," Applicable Analysis: An International Journal, vol. 69, no. 1-2, pp. 149-156, 1998.

[13] K. Omrani, F. Abidi, T. Achouri, and N. Khiaria, "A new conservative finite difference scheme for the Rosenau equation," Applied Mathematics and Computation, vol. 201, no. 1-2, pp. 3543, 2008.

[14] B. Feng, X. C. Min, Y. Y. Yu, and B. Hu, "A new three-level conservative finite difference scheme for the Rosenau equation," Journal of Sichuan University. Natural Science Edition. Sichuan Daxue Xuebao. Ziran Kexue Ban, vol. 48, no. 1, pp. 7-12, 2011.
[15] S. A. V. Manickam, A. K. Pani, and S. K. Chung, "A second-order splitting combined with orthogonal cubic spline collocation method for the Rosenau equation," Numerical Methods for Partial Differential Equations, vol. 14, no. 6, pp. 695-716, 1998.

[16] D. Gilbarg and N. Trudinger, Elliptic Partial Differential Equations of Second Order, Springer, Berlin, Germany, 1977.

[17] A. Quarteroni, A. M. Quarteroni, and A. Valli, Numerical Approximation of Partial Differential Equations, vol. 23, Springer, 2008.

[18] J. Zhang and C. Xu, "Finite difference/spectral approximations to a water wave model with a nonlocal viscous term," Applied Mathematical Modelling: Simulation and Computation for Engineering and Environmental Systems, vol. 38, no. 19-20, pp. 49124925, 2014. 


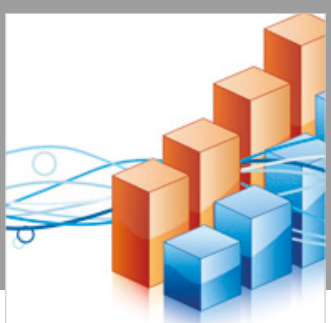

Advances in

Operations Research

\section{-n-m}
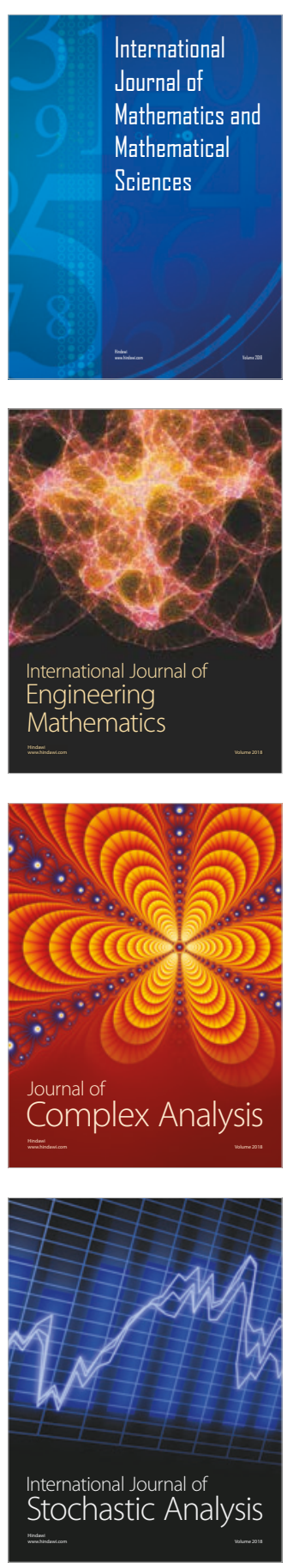
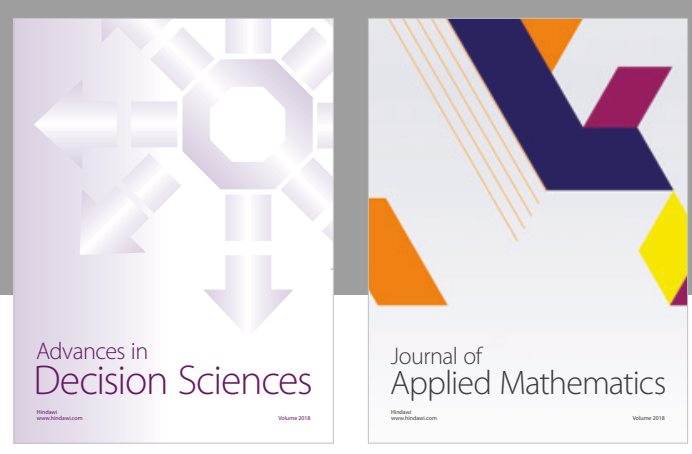

Journal of

Applied Mathematics
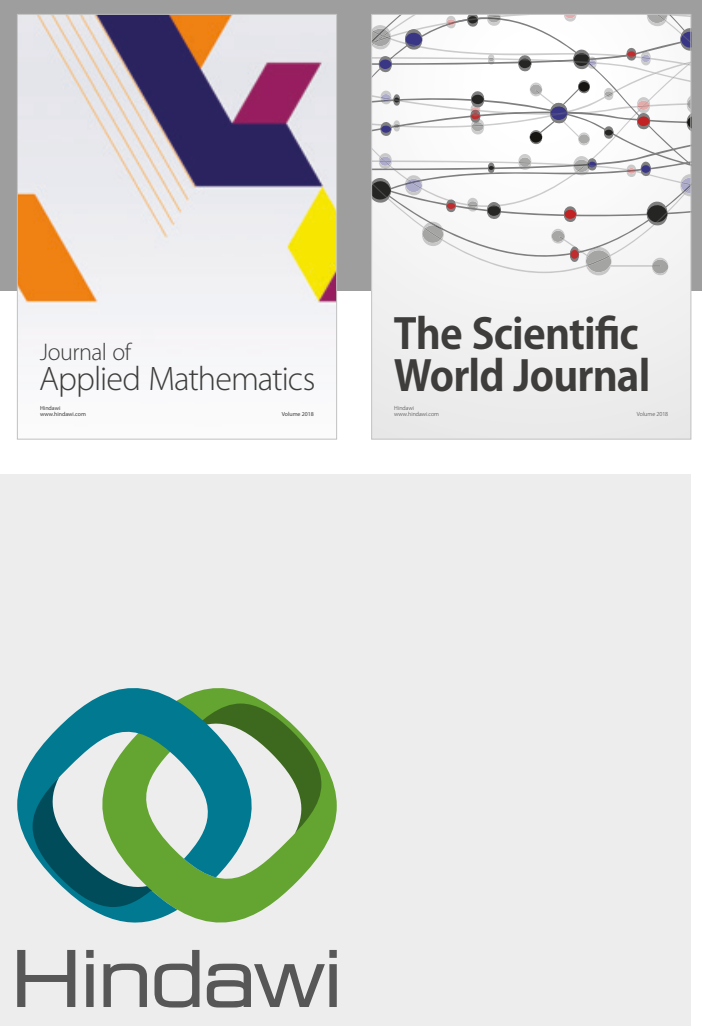

Submit your manuscripts at

www.hindawi.com

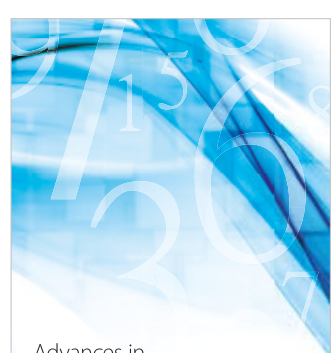

Advances in
Numerical Analysis
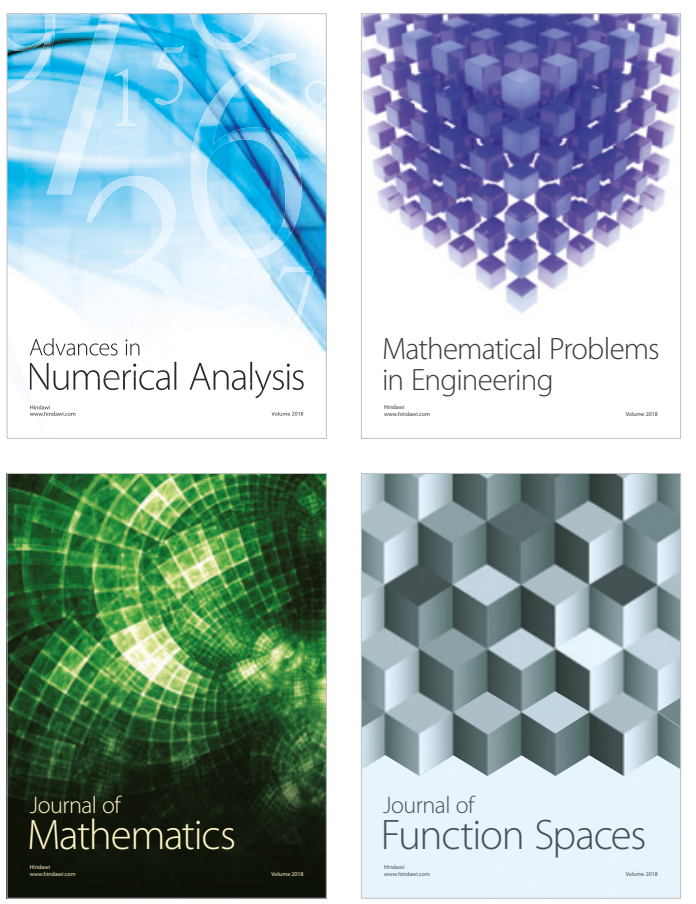

Mathematical Problems in Engineering

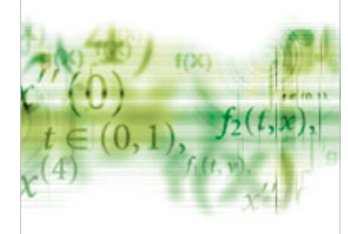

International Journal of

Differential Equations

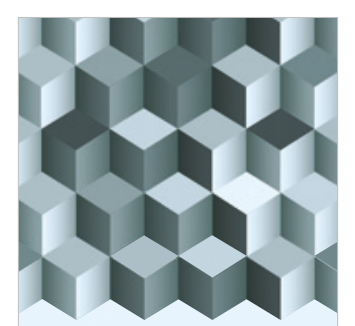

Journal of

Function Spaces
The Scientific

World Journal

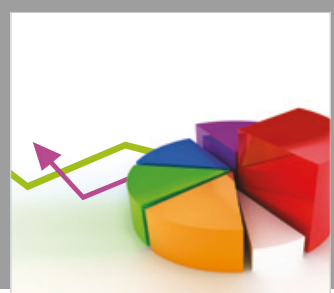

Journal of

Probability and Statistics
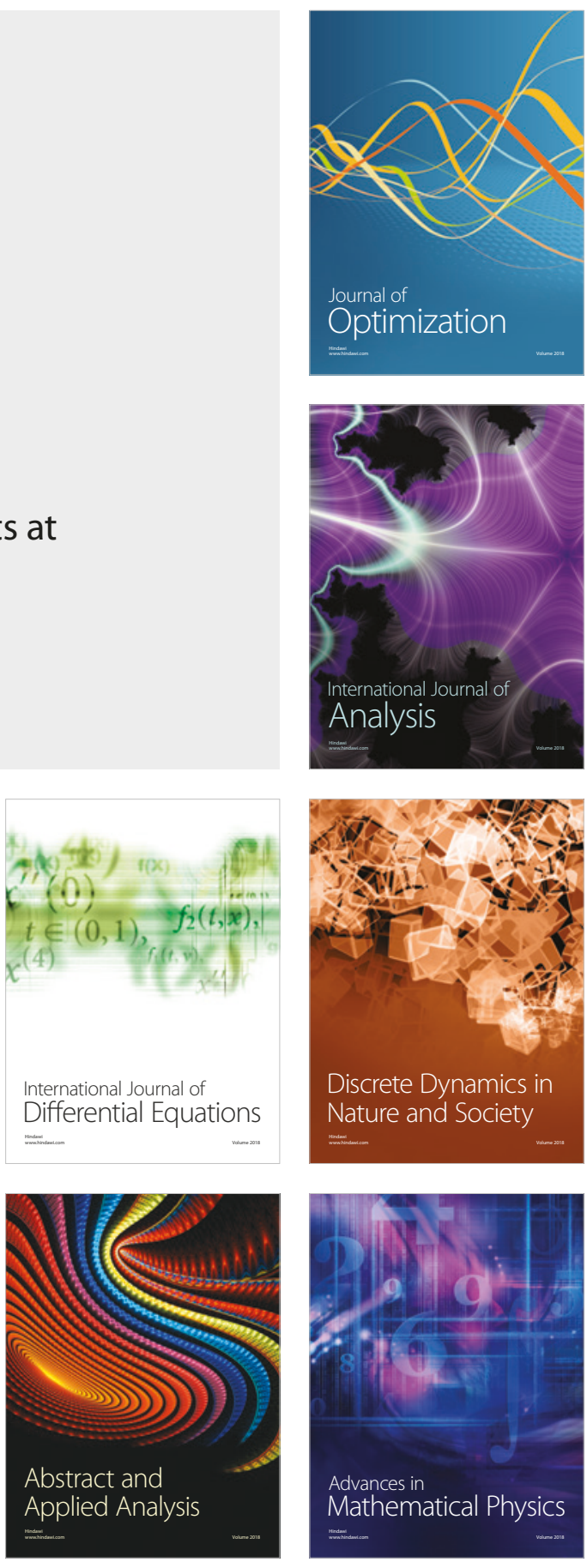